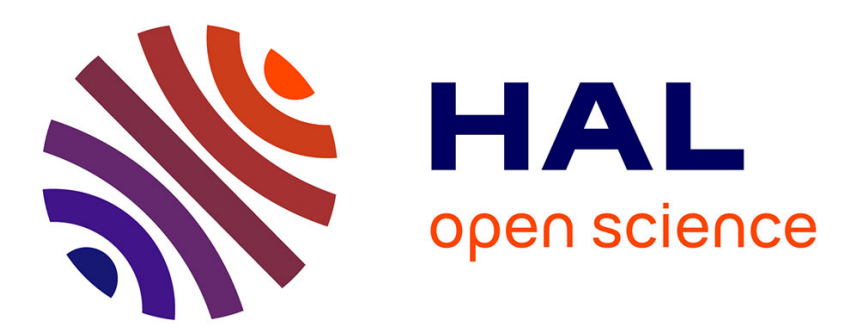

\title{
Les métamorphoses du nom propre modifié entre le français et le russe \\ Evgeny Shokhenmayer
}

\section{To cite this version:}

Evgeny Shokhenmayer. Les métamorphoses du nom propre modifié entre le français et le russe.

Langues et textes en contraste, Sep 2009, Växjö, Suède. pp.127-143. halshs-00530817

\section{HAL Id: halshs-00530817 https://shs.hal.science/halshs-00530817}

Submitted on 29 Oct 2010

HAL is a multi-disciplinary open access archive for the deposit and dissemination of scientific research documents, whether they are published or not. The documents may come from teaching and research institutions in France or abroad, or from public or private research centers.
L'archive ouverte pluridisciplinaire HAL, est destinée au dépôt et à la diffusion de documents scientifiques de niveau recherche, publiés ou non, émanant des établissements d'enseignement et de recherche français ou étrangers, des laboratoires publics ou privés. 


\title{
Les métamorphoses du nom propre modifié entre le français et le russe ${ }^{1}$
}

\author{
Evgeny Shokhenmayer ${ }^{1}$ \\ ${ }^{1}$ Laboratoire MoDyCo, Chercheur associé \\ Université Paris Ouest Nanterre La Défense, \\ eugen1979@mail.be \\ Kingsleyallee 8A, 32425 Minden, Allemagne
}

\begin{abstract}
Biographie: Le 28 avril 2009 j'ai passé ma soutenance de thèse avec summa cum laude, après avoir effectué 4 ans de recherches („Champs associatifs des noms propres et mécanismes de la compréhension textuelle“) dans le laboratoire MoDyCo, à l'École Doctorale «Connaissance, langage, modélisation », sous l'égide de l'Université Paris Ouest Nanterre La Défense. J'ai traduit, enseigné et fait des recherches des langues étrangères pendant 2 ans en Russie, 4 ans en France et 2 ans en Allemagne. À l'heure actuelle, je travaille en tant que professeur des langues (Français, Latin, Anglais et Russe) à Gesamtschule Minden (Allemagne, NRW).
\end{abstract}

\section{Introduction}

Au départ nous décodons brièvement ce que nous entendons sous le terme «nom propre modifié », qui est pourtant assez connu et fort discuté. La discussion concernant la modification du nom propre (désormais Npr) suscite un intérêt très spécial [p.ex., S. Leroy « Noms propres : la modification », Langue française, 2005]. G. Kleiber a inspiré la typologie des emplois modifiés (dénominatif, exemplaire, métonymique, etc.) et francisé le terme de Tyler Burge «modified proper name $»^{2}$. Cette contribution a pour objet la présentation des contrastes entre les métamorphoses des noms propres référentiellement / sémantiquement et/ou morphologiquement / syntaxiquement modifiés.

Les Npr peuvent subir tout un éventail de modifications : grammaticalisation, verbalisation, adjectivation, adverbialisation, métaphorisation, métonymisation, communisation, généralisation, particularisation, dépropriation, désemantisation, appellativisation, perte de majuscule, affixation, flexion, forme diminutive, forme hypocoristique, etc. Lorsqu'on parle des Npr modifié, le plus souvent il s'agit de l'emploi des unités propriales avec les articles, déterminants, subordonnées, attributs, appositions, au pluriel et sans majuscule. Quelques constructions avec article poussent le nom propre vers une certaine «communisation», tandis que d'autres constructions lui conservent son statut. Comment expliquer cette différence?

Beaucoup de théoriciens y voient des processus différents et inventent de nouveaux termes pour qualifier ce phénomène et nommer ses formations consécutives :

\footnotetext{
Rhétorique et philosophie antique : éponyme/antonomase

1660 Arnauld et Lancelot : Npr - noms généraux - Npr «par figure »

1767 Beauzée : Npr - appellatifs

1798 Condillac : Npr « figurement » employé

1935 G. et R. Le Bidois : Npr employé «par emphase » ou « au figuré »

1950 Damourette et Pichon : Npr « communeusement » employé
}

\footnotetext{
${ }^{1}$ Metamorphoses of Modified Proper Name between French and Russian // Les cahiers 13-14 of 3èmes Journées francosuédoises de linguistique «Langues et textes en contraste » by Sens public, Växjö University, Sweden, 28-30th September 2009, p. 127-144, published on July 2010. (in French).

2 (1973) Reference and Proper Names, The Journal of Philosophy, LXX, 70: 425- 439.
} 
1954 Gardiner : common proper names - (dis)embodied proper names / Npr incarné

1954 Pottier : surnoms

1958 Lévi-Strauss : mythème = mythologème onomastique

1961 Jespersen : quasi proper names

1961 Shwayder : noms propres usagés

1968 Zabeeh : used proper names

1973 Algeo : primery - secondary proper names

1973 Pariente : noms propres épistémiques

1973 Burge : modified proper name

1980 Otin (rus.) : connotonyme = onyme connotatif

1980 Grodziński : quasi-ideal proper names - multi-designatory names

1981 Meyer et Balayn : Npr quidditif

1985 Langendonck : appellative derived

1987 Kara'ulov / Gudkov (rus) : Npr de précédent / Npr intertextuel

1989 Blokh et Semenova (rus) : onyme semi-commun ou semi-onyme

1990 Kaplan : generic names - common currency names

1990 Neroznak (rus) : appellonyme = appellatif + onyme

1991 Flaux : Npa (nom propre en antonomase)

1992 Künne : hybrid proper names

1992 Schweickard (all) : Deonym

1993 Likhačev (rus) : Npr - concept

1994 Jonasson/Gary-Prieur : Npr exemplaire

1996 Forest : idionymes directs - indirects

1997 Wilmet : Npr essentiels - Nc accidentels

1999 Hadj Hamou : proper common nouns

1999 Kalinkin (rus) : poétonyme = onyme «poétique »

2000 Fomin (rus) : réminonyme = onyme de réminiscence

2001 Katz : improper names

2001 Boulanger et Cormier : proprionymes

2004 Chekhtman / Zakharova (rus) : allonyme = nom propre allusif

\section{Unité propriale modifiée ou ex-propriale}

La multitude des termes prouve que la frontière entre $\mathrm{Npr}$ et nom commun (désormais $\mathrm{Nc}$ ) est délicate à définir. Les Npr modifiés sont tellement hétérogènes qu'on ne peut pas créer une théorie uniformiste. Une telle théorie résoudrait en effet de manière séduisante le problème posé par l'existence, à côté des emplois canoniques, non modifiés, des $\mathrm{Npr}$ dits modifiés. Les onomasticiens doivent soit assimiler au Nc le Npr déterminé, soit imaginer un dispositif de passage spécial du Npr non articulé au Npr articulé.

L'appellation la plus usuelle en linguistique française est antonomase $d u \mathrm{Npr}$ : il s'agit d'un trope, d'une figure rhétorique en discours, qui s'applique aussi aux Npr modifiés (un nouveau Mozart, les Napoléons de demain, etc.). Déonyme serait acceptable, mais ce terme 
désigne toutes les formes dérivées d'un $\mathrm{Npr}$, ce qui est trop général. On peut de même écarter l'étiquette $\mathrm{Npr}$ modifié, qui est en quelque sorte le terme «fourre-tout». On pourrait retenir la dénomination du Npr lexicalisée ou encore celle de Npr substantivé.

La nécessité d'étudier en détail les formes onymiques avec déterminant est également justifiée par le fait que certains chercheurs rangent d'une façon indifférenciée toutes ces formes dans la classe des appellatifs dérivés (appellative derived de W. Van Langendonck). Ainsi, on rattache à l'antonomase lexicalisée non seulement les dérivations sémantiques et lexicales des noms personnels, mais aussi les mêmes anthroponymes nommant différentes personnes, membres de la famille y compris ; les nominations du même individu se rapportant à diverses périodes temporelles, états mentales et psychologiques ; les Npr remontant aux prototypes, et les anthroponymes modifiés par des attributs limitatifs ${ }^{3}$. Les emplois articulés onymiques sont semblablement interprétés par Curme [1955], Langendonck [1985] et autres. Cependant, on peut estimer que les formes articulées ressemblent aux Nc uniquement du point de vue fonctionnel, sans perdre leur essence anthroponymique [Superanskaja, 1973; Boër, 1978]. L'article (actualisateur, déterminant, quantifiant ou encore spécificateur) détermine le quantum de l'extension en discours.

Pour Noailly [1987 : 77], «l'usage libre des articles devant le Npr fait de celui-ci un terme classificateur. Quand je dis un Alfred Muller, quel que soit l'effet rhétorique de l'article (...), je pose l'existence d'une classe d'Alfred Muller. Au contraire, quand je dis Emile, je ne pose pas du tout l'existence potentielle d'une classe d'Emile. Je désigne, c'est tout ». Que deviennent les Npr "modifiés", c'est-à-dire ceux qui, comme les Nc, prennent des déterminants ? La position kleibérienne est nette, qui considère la plupart d'entre eux comme des prédicats, c'est-à-dire des termes généraux, des $\mathrm{Nc}$ en quelque sorte, qui dénotent une certaine catégorie regroupant des occurrences sur la base de propriétés communes. Ce contenu descriptif a une différence de taille, toutefois, par rapport à celui des Nc standards : il ne s'agit pas de catégories conventionnelles, lexicalisées. Tous ces emplois de Npr modifiés, ainsi que l'ont montré les études de Gary-Prieur et Jonasson, ne peuvent s'expliquer ou s'interpréter qu'à partir du Npr non modifié. Ce sont donc des Nc "discursifs", dérivés des emplois prédicatifs ou descriptifs de formes normalement non destinées à une telle tâche. La diversité des emplois relevés n'est qu'une conséquence de ce caractère non lexical, et leur interprétation doit s'effectuer à partir du Npr source [Kleiber, 1994 : 32].

Les Npr référentiellement modifiés ont une valence sémantique ouverte au déterminant qualitatif, et sont facilement associés avec les syntagmes attributifs, qui exercent une influence pragmatique sur le destinataire de l'information, et mettent en évidence la perception subjective du monde par le destinateur. Liés à la réalisation pragmatique de l'intention de l'émetteur, les Npr polyréférentiels attributivement développés reflètent un conditionnement présuppositionnel et situationnel de l'énoncé, en indiquant les propriétés du sujet parlant, telles que position sociale, thésaurus personnel, particularités psychophysiologiques, intérêt à la réussite de la communication.

\footnotetext{
${ }^{3}$ Vendler, $1967: 120$; Kałuza, $1981: 17-19$.
} 
Les spécificités sémantico-fonctionnelles des Npr acquièrent, en français/anglais et en russe, un marquage différent à cause des caractéristiques constructionnelles de ces langues. En français, le Npr polyréférentiel coopère avec tout le paradigme des articles (défini, indéfini et zéro). La déterminabilité du Npr par l'article zéro à valeur d'abstraction absolue est due à la «fission» référentielle (referential split) de l'onyme: avec le $\mathrm{Npr}$ pris au figuré, la caractérisation est orientée vers l'individu pour lequel sa fonction identifiante se trouve nonsignificative.

L'existence du système des articles dans les langues française et anglaise, qui distinguent les Npr modifiés, transforme l'antonomase en procédé stylistique plus souple et indépendant du complément attributif, par comparaison avec les corrélats russes. Puisque les articles jouent un rôle sémantico-formatif, causant la recatégorisation de l'anthroponyme, leur présence seule dans les nominations syntaxiquement non-développées est suffisante pour expliquer le sens caractérisant onymique. Dans la langue russe, faute d'articles, la fonction des explicateurs de figurativité est réalisée par différents indicateurs contextuels et, premièrement, par les attributs. Notre corpus justifie qu'en russe on utilise de préférence les nominations attributivement développées, qui sont construites selon le principe du désaccord sémantique entre l'attributmodificateur et le sens du Npr (sens primitif). La dissonance sémantique du déterminant et du déterminé, en détruisant la monoréférentialité de l'anthroponyme, facilite le décodage de l'image par le destinataire.

Quant à l'usage des Npr stylistiquement marqués, l'onomastique note que les anthroponymes métaphoriques permettent des emplois articulés, aussi bien que non-articulés, mais, en règle générale, ils s'associent à l'article ${ }^{4}$.

Les chercheurs francophones, dans le cadre référentialiste et syntaxique, mentionnent que, d'une part, le «déterminant n'entraîne pas toujours une modification sémantique » [Jonasson, 1994 : 12] et, d'autre part, le Npr «employé sans déterminant ne désigne pas forcément son référent initial » [Gary-Prieur, 1994 : 35]. Il n’y a pas donc de critères précis pour déterminer ces emplois divers. Nous ajouterons que la première considère qu'employé sans article, le Npr réfère sans recourir à un sens, c'est «un désignateur direct et rigide» [Jonasson, 1990 : 123]. La deuxième pense que «la possibilité d'une interprétation métaphorique est inscrite dans la définition même du nom propre » [Gary-Prieur, $1994: 81]$.

Sans expliciter la différence entre «Je suis Achille» et «Je suis un Achille », Meyer et Balayn jugent identiques le signifié métaphorique et le signifié antonomasique [1981: 192]. Le rôle des articles, en tant que marqueurs sémantico-fonctionnels nucléaires des noms personnels français transposés, peut être précisé si l'on compare le fonctionnement des Npr en français (langue à articles) et en russe (langue sans articles). La catégorie «déterminabilité indéterminabilité » dans la langue russe porte le caractère « masqué » (terme de B.L. Whorf), vu que l'individualisation, l'identification et la généralisation des objets désignés s'effectuent contextuellement et complexement. En particulier, en russe, pour inclure la catégorie

\footnotetext{
${ }^{4}$ Searle, 1969 : 135 ; Burge, 1973 : 429 ; Superanskaja, 1973 : 119 ; Boër, 1978 : 76-77, 99.
} 
« déterminabilité - indéterminabilité » dans la perspective sémantique du texte, il faut activer d'autres catégories, celle de nombre, de cas, de degrés de comparaison.

L'article n'est qu'un des moyens traduisant la vaste catégorie de la 'détermination indétermination'. Si nous considérons uniquement les articles, nous ne pouvons étudier qu'une seule partie de cette problématique. Il est insuffisant de tenir compte seulement des articles lorsqu'on analyse les déterminants du Npr, car d'autres éléments déterminatifs entrent en jeu :

1) unités «communicatives »: pronoms, adjectifs, constructions descriptives, subordonnées particulières ;

2) désinences de $\operatorname{cas}^{5}$;

3) numéral «un(e)»;

4) indices implicites (contextuels);

5) ordre de mots ;

6) intonation [Feoktistov, 1969].

Les ouvrages qui parlent de la problématique anthroponymique et du fonctionnement des articles, traitent presque toujours la question des rapports de l'onyme à la catégorie de l'(in)détermination. Or, les études sur la grammaire du $\mathrm{Npr}$ amènent les linguistes à des conclusions différentes, sinon contradictoires.

\section{Unité propriale déterminée}

«On lit constamment que le nom de personne s'utilise sans déterminant, mais il n'est pas nécessaire de faire référence [aux] langues exotiques pour prouver le contraire. » [Vaxelaire, 2005 : 84]. C'est le cas en russe, où le système d'articles est absent, et partiellement en français, où il y a l'habitude de distinguer, assez strictement selon Dubois, les Npr «qui admettent l'article défini » et «se comportent comme les substantifs», et «ceux qui n'admettent pas l'article » et «se comportent comme des prénoms » [1965: 155]. Presque tous les linguistes expriment l'idée que le Npr ne peut pas être accompagné d'un déterminant parce qu'il est «parfaitement déterminé », « essentiellement notoire » [Damourette et Pichon, 1911-1927: 520 ] et «particulier à un être » [Brunot, $1965: 39]$.

Une vision plus claire des articles, comme marqueurs de la spécialisation fonctionnelle des Npr français polyréférentiels, peut être atteinte si l'on rattache cette problématique à la question générale du fonctionnement déterminé des unités linguistiques stylistiquement marquées, dans les langues française/anglaise (articulées) et russe (non-articulée).

On refuse ordinairement les déterminants suivants :

Article défini (Chomsky [1975], Sloat [1969], jeune Kleiber [1981 : 306]) :

Pourtant M. Noailly montre [1999: 93] que l'article est obligatoire avec l'épithète, mais non pas avec les attributs de l'objet. Il est étonnant que pour Droste [1975 : 9], le déterminant adjectival fait automatiquement de l'onyme un Nc. Selon R. Martin, le Npr «n'admet pas

\footnotetext{
${ }^{5}$ Pour les langues où il y en a, comme le russe, par exemple.
} 
l'usage « générique » de l'article le : la valeur « intensionnelle » de celui-ci s'y oppose » [1987 : 147].

Les SN complémentés par les attributs identifiants possèdent sans doute une potentialité connotative différente. Ainsi, les Npr modifiés par les adjectifs de titre connotent un trait social (Le Révérend George Melchisedeck Watson). Les autres identificateurs réalisent dans l'énoncé les connotations factuelles, qui dépendent de la situation des référents onymiques (l'ancienne Mary Chisholm, le premier Mrs. Todd). Toutes ces nuances de sens discursifs trouvent aussi leur équivalent en russe, mais ce sont uniquement les attributs et les déterminants qui se chargent de découvrir la spécificité sémantico-fonctionnelle: le Révérend John Wesley Caldwell I преподобный Джон Уесли Колдуэлл; the out-of-coffee Margie / Утренняя Марджи, приходившая в лавку за кофе.

Les attributs qui indiquent un trait individuel marquant, en français, sont en général exprimés par des adjectifs antéposés. En russe, on peut employer des substantifs apposés: le très beau Lazarus / красавчик Лазарус ${ }^{7}$; le pauvre Hastings / бедняга Гастингс ${ }^{8}$.

L'«expansion à droite du Npr » est possible [Gary-Prieur, 1994: 148], elle peut être adjectif descriptif (exemplaire) ou restrictif (métaphorique) [Leroy, $2005: 88$ ] et « renforce les ambiguiités » [ibid.]. Parfois l'expansion à droite sert non à préciser ou identifier le Npr modifié, mais à le recatégoriser d'une façon allégorique, à le reclassifier, à l'incorporer dans un contexte absolument inhabituel (les Napoléon de demain) où se mélangent l'interprétation exemplaire et l'identification du référent-cible. C'est pour cela qu'«on trouve d'ailleurs parfois des constructions comparatives dans lesquelles la présence d'une expansion à droite du Npr oriente encore davantage vers une interprétation métaphorique ...» [ibid. : 95]. L'expansion à gauche est préférable en russe s'il s'agit des constructions attributives avec épithètes, car la grammaire permet l'attribution antécédente (словно заумный Рабле ${ }^{9}$ ). L'expansion à gauche ou à droite est un contexte immédiat qui suppose très souvent la recatégorisation et la restriction, donc l'identification qualitative.

Le matériel analysé témoigne de ce que les anthroponymes identificateurs sont rarement utilisés sans expansion. Lorsque le détachement net du référent est présenté sans modifieur attributif, à part l'article défini, la fonction identifiante «hypertrophiée » du Npr modifié est complètement déterminée par le cotexte, ou plus souvent, par le contexte de tout une œuvre.

Article indéfini ([Pulgram, $1954: 42]$ )

C'est le cas le plus problématique, quand on explicite la compatibilité d'un article indéfini et du Npr bien défini. Pour Noailly [1987 : 77], cela fait d'un Npr un Nc ; pour Pamp [1985 : 112], cette interprétation n'est pas acceptable puisque le Npr est monoréférentiel. L'exemple J'avais rencontré un Boutonnet est interprété soit par le prédicat de dénomination kleibérien (être appelé $N$ ), soit par l'appartenance à une famille, mais il y a des combinaisons qui

\footnotetext{
${ }^{6}$ la Margie matinale, étant allée chercher du café dans la boutique (trad. Y. S.).

${ }^{7}$ le petit-maître Lazare (trad. Y. S.).

${ }^{8}$ le pauvre hère Hastings (trad. Y. S.).

${ }^{9}$ comme Rabelais hermétique.
} 
« exigent »d'autres approches : celles dites de parangon et de fractionnement ne changent pas référentiellement le statut du $\mathrm{Npr}$, mais elles l'aspectualisent et le spécifient.

Le caractère «modifié », assuré par l'article indéfini (critère syntaxique), est « rendu problématique par la référence effectuée à son porteur initial » (critère référentiel) [Leroy, 2005 : 86]. C'est pourquoi ces $\mathrm{Npr}$ «restent confinés dans leur essence primitive » [Damourette et Pichon, 1968 : 523-524] et peuvent être considérés comme «à la fois un Npr modifié et Npr non modifié » [Jonasson, 1994 : 232]. Les explications sont majoritairement d'ordre référentiel ${ }^{10}$, c'est-à-dire que le Npr en emploi exemplaire renvoie à sont référent initial et à lui seul, tandis que le Npr en emploi métaphorique renvoie à un référent autre.

Comme en russe les articles sont absents, le sens discursif de l'anthroponyme de supposition se constitue à l'aide des explicateurs lexicaux avec une tendance correspondante hypothétiquement nuancée. Comparez:

Margie nous attendait déjà, toute l'incarnation de l'hospitalité. Elle nous a présenté son compagnon, un M. Hartog de New-York, dont le visage était bronzé par la lampe à quartz et la bouche était tellement planté avec des dents qu'elle ressemblait à l'épi de maïs. Monsieur Hartog avait l'air d'être emballé et enveloppé en cellophane et à chaque réplique il répondait par un rire approbatif. [J. Steinbeck, L’hiver de notre mécontentement, 1979 : $161]$

et

Марджи уже ждала нас, вся - воплощение гостеприимства. Она представила нам своего кавалера, некоего мистера Хартога из Нью-Йорка, у которого лиџо было покрыто загаром, приобретенный под квариевой лампой, а рот так тесно усажен зубами, что напоминал кукурузный початок. Мистер Хартог казался хорошо упакованным и завернутым в целлофан и на любое замечание отвечал одобрительным смехом [Дж.Стейнбек, Зима тревоги намей, 1985 : 535]

On voit que la connotation de l'hypothétique, subjective par nature, dépend du rapport sujet-objet et du degré de catégoricité, ce qui conditionne son inclusion dans le cadre modal de l'énonciation. Par suite, son actualisation s'effectue d'habitude parallèlement avec la translation, par le biais des termes modaux, des significations syntaxiques selon les catégories «rapport modal du sujet à l'action » et « rapport sujet - objet ».

En français, les anthroponymes de restriction virtuelle s'emploient avec ou sans complémentation, mais en russe ce type de Npr est toujours accompagné d'un GN. De fait, dans les langues slaves, la détermination du nom personnel par un GN qualifiant est considérée comme l'indice linguistique principal, sinon unique, de la bifurcation sémiotique d'un onyme. Les déterminatifs peuvent corollairement servir d'indicateur de la fonction pseudo-qualifiante, car ils actualisent la sémantique de la généralisation relative. Comparez :

\footnotetext{
${ }^{10}$ Meyer et Balayn [1981 : 187], Gary-Prieur [1994: 144-146], Jonasson [1994 : 232-233], Flaux [2000 : 137139], Leroy [2005: 86-88]), Vaxelaire [2005 : 115-117].
} 
Le premier tiers de l'album - Angelo montrait d'abord ces photos-là - se dévouait à l'enfance d'Angelo, un jeune Angelo_vivait avec une grande famille sur l'avenue Atlantic dans Brooklyn, - croyez-vous ou non, mais regarder, voilà le soldat qui a vraiment une véritable famille, la voilà toute, tous les quinze membres... [J.Jones, Tant qu'il y aura des hommes, $1951: 190]$

et

Первая треть альбома - Анджело обязательно показывал сначала эти снимки - посвящалась детству Анджело, тому Анджело, который жил с большой семьей на Атлантик-авеню в Бруклине, - не верите, смотрите сами, вот вам солдат, у которого и вправду есть настоящая семья, вот она вся, все пятнадиать человек... [Д. Джонс, Отныне и вовек, 1986 : 209].

De plus, comme la dominante sémantico-fonctionnelle dans les nominations pseudoqualifiantes russes est notamment exprimée par l'attribut, on observe souvent l'omission du Npr lui-même dans la traduction.

Dans la langue russe, la transposition du Npr en anthroponyme modifié de restriction réelle s'effectue uniquement au moyen du contexte explicite qui montre les traits essentiels du porteur d'un nom en tant que représentant d'un certain collectif. Comparez :

... elle est une Cutrere... [T. Williams, La descente d'Orphée, 1961 : 232].

et

... её фамилия Катрир... [Т. Уильямс, Орфей спускается в ад, 1962 : 12-13].

Bien que les marqueurs formels des Npr polyréférentiels soient absents, l'antonomase russe est facilement reconnue par le lecteur à cause du caractère paradoxal du sens discursif réalisé :

Несмотря на то, что год всегда новый, встречают его одинаково. Елка с подарками, шампанское с салатом Оливье, очередной Путин в телевизоре. Говорят, традииии. ${ }^{11}$ [Аргументы и Факты, 24/12/2003]

Ельцины приходят и уходят, а идея нама навсегда $a^{12}$. [Маркетинг и Консалтинг, 17/02/2003]

На "Трех мушкетерах" читаю: "Дорогому Бухарину - Портосу первой пятилетки. Не надо враждовать с гвардейцами Ришелье. И. Сталин". ${ }^{13}$ [Огонек, 1990. № 41 : 12]

\footnotetext{
${ }^{11}$ Malgré ce que l'an est toujours nouveau, on le rencontre de la même manière. Sapin avec cadeaux, champagne avec salade Olivier, un Poutine suivant à la télé. On dit : les traditions (traduction de Y. Sch.).

${ }^{12}$ Les Eltsine vont et viennent, mais notre idée est pour toujours (trad. Y. S.).

${ }^{13}$ Sur « Les trios mousquetaires » je lis : « À cher Boukharine - à Portos de la première période quinquennale. Il ne faut pas être en mauvais rapports avec les officiers de la garde de Richelieu. » J. Staline (trad. Y. S.).
} 
Donc, les exemples cités montrent que les probabilités d'interpréter différemment l'antonomase-synergie sont restreintes par le référent antonomasique, qui forme le contexte nécessaire à la compréhension de la nomination métaphorique.

Dans la langue russe, à cause de l'absence du système d'articles, le repérage de la sémantique qualificative et généralisante du Npr au figuré s'effectue par le biais du contexte explicatif (pronoms indéfinis, attributs) qui abolit la monoréférentialité onymique, mais aussi moyennant l'usage du pluriel. Il est intéressant que les indicateurs de la bifurcation double de signe (multiplication) se présentent dans différentes combinaisons :

Et comme, tout naturellement, l'assentiment $d u$ plus grand nombre, les applaudissements, le succès, les faveurs, vont à ce que le public peut aussitôt reconnaître et approuver, c'est-à-dire au conformisme, je me demande avec inquiétude si, peut-être, dans l'U.R.S.S. glorieuse d'aujourd'hui, ne végète pas, ignoré de la foule, quelque Baudelaire, quelque Keats ou quelque Rimbaud qui, en raison même de sa valeur, a du mal à se faire entendre. Et c'est pourtant celui-là entre tous qui m'importe, car ce sont les dédaignés de

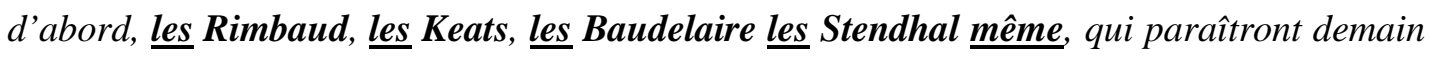
les plus grand. [GIDE A., Retour de l'URSS, 1936 : 98].

et sa traduction en russe :

И поскольку (это само собой разумеется) благосклонности, аплодисментов большинства удостаивается все то, что публика тотчас может признать и одобрить, то есть то, что порождено конформизмом, я с беспокойством спрашиваю себя : что, если в славном ныне Советском Союзе прозябает неведомый толпе какой-нибудь Бодлер, какой-нибудь Китс, или какой-нибудь Рембо, и он, этот избранник, не может заставить усльшать себя. Но именно он, единственный из всех, мне важен и интересен, ибо отверженные сначала - Рембо, Китсы, Бодлеры, Стендалй даже - завтра станут великими [Жид А., Возвращение в СССР, 1990 : 96].

L'interprétation exemplaire (parangon ${ }^{14}$ ) concerne « un Npr n'admettant aucune expansion » [Gary-Prieur, $1995: 247]$ et « désigne un référent bien connu dans la communauté linguistique » [Jonasson, 1994 : 229]. On estime possible, dans ces cas, de supprimer l'article sans que cela ne change le sens de l'énoncé, le Npr continuant de désigner son référent «initial » [Gary-Prieur, 1994 : 146-149, Vaxelaire, 2005 : 116]. Si c'est correct, à quoi bon introduire cet article indéfini ? Quelle est la motivation de l'émetteur? La multiplication des référents paraît aussi très épineuse (des Berlusconi) : si la suppression de l'article ne change rien, cela porte sur

\footnotetext{
${ }^{14}$ L'emploi exemplaire du Npr est étudié par Meyer et Balayn [1981:187-188], qui parlent d'emploi quidditif, par Gary-Prieur [1994 : 134-149, 1995 : 247-248] - interprétation un Npr $\varnothing$, par Jonasson [1994 : 229-233], Flaux [2000b : 137-139], Leroy [2005 : 84-98] - emploi exemplaire.
} 
la création d'une classe virtuelle de personnes aux qualités semblables. Donc, c'est la catégorie qualitative qui est activée et non pas la propritude en tant que telle. Pour Vaxelaire, ce n'est qu'un «effet stylistique » [ibid.].

En outre, le statut du Npr modifié en emploi exemplaire est douteux pour certains linguistes [Jonasson, 1994: 232 ; Leroy, 2005 : 86], qui soulignent la contradiction entre le critère syntaxique (article indéfini) et le critère référentiel (référent initial du Npr). Il nous semble que la référence effectuée peut porter bel et bien sur le porteur initial ou actuel du Npr, mais peut se focaliser sur quelques propriétés importantes au moment de l'énonciation, du point de vue de l'émetteur. Il s'agit des déplacements «subtils » du focus de l'attention : celui-ci s'aiguille non pas dans une voie nouvelle (une essence nouvelle), mais il se fixe sur une des qualités du référent même. Ce fait est confirmé par la psycholinguistique du point de vue de la structure de l'image [Vasiljuk, 1993 : 19] : « Сознание может быть направлено на предмет, но в то же время сфокусировано не на самом предмете, но на впечатлении от предмета ${ }^{15} \gg$. Donc, les différents degrés de motivation (critère cognitif), les divers focus de la perception dans le cadre énonciatif ou textuel (critère référentiel) provoquent les modifications de la forme linguistique (critère syntaxico-morphologique).

L'interprétation de fractionnement présente un «instant» ou une «facette » des personnes qui portent les noms en question. Cette compatibilité de l'article indéfini (critère syntaxique) avec le Npr défini (critère référentiel) peut «prêter à confusion puisque le référent y est à chaque fois bien connu » [Vaxelaire, 2005 : 117]. Pour Kleiber, ce sont des « occurrences de particuliers » [1996: 580], pour Wilmet, des «avatars du nom propre » [1986:44-45]. Du point de vue de la sémantique, Vaxelaire ne différencie pas un Victor Hugo débordant d'énergie et le Victor Hugo poète: pour lui [2005 : 118], comme pour Gary-Prieur, ce sont des Npr car « la pluralisation ne peut pas s'expliquer par un changement de catégories » [1996:138], bien que Bonnard [2001 : 33] parlent ici de Nc. Allerton les considère en tant que Npr dérivés [1987 : 66] ce qui est inacceptable à cause de l'instabilité du signe où le processus de figement a rarement lieu.

\section{Démonstratif [Pulgram, 1954 : 42]}

D'après G. Kleiber, il existe trois types d'interprétations possibles des démonstratifs devant les $\mathrm{Npr}$ : dénominative, distanciée et affective. Il faut noter le caractère paradoxal d'une combinaison suivante: la «déterminabilité double» attendue (déterminant + Npr) devient en réalité une «déterminabilité insuffisante ». Ils remarquent que cette construction est souvent employée lorsque le sujet parlant ne connaît pas personnellement le référent du nom. La question Qui est Paul? appelle une précision sur la profession ou la nationalité (cf. What is Paul ?), mais Qui est ce Paul? présuppose à la fois la précision mentionnée ci-dessus et l'identification, quand le locuteur veut faire le lien entre le Npr et une personne (cf. Who is Paul ?).

\footnotetext{
15 «La cognition peut être orientée vers l'objet, en étant focalisée non pas sur l'objet même, mais sur l'effet produit par l'objet ». (trad. Y. S.)
} 
De plus, cette combinaison est possible lorsque l'émetteur ne doute pas de la nomination mais de sa pertinence, ou qu'il veut exprimer une attitude négative (péjorative) : c'est ce que nous voyons dans l' «effet de distanciation » kleibérien. Il est important de rappeler que le démonstratif «ce» a une signification non seulement déictique mais aussi anaphorique (métatextuelle), sauf dans les cas où cette construction ouvre le texte. Vaxelaire affirme que la « compatibilité avec les démonstratifs et les possessifs est le résultat d'un effet stylistique et ne change en rien les noms propres, car ils désignent toujours les mêmes individus » [2005 : 113].

En ce qui concerne l'article défini et démonstratif, leur différenciation dépend de la spécialisation fonctionnelle propre à chacun des corrélats. La valeur essentielle de l'article défini consiste à individualiser l'objet servant de la base à l'identification ; celle du démonstratif est l'identification même par corrélation du Npr et du référent, soit d'une façon directe (la deixis prend appui sur la situation d'un acte discursif), soit indirectement, par la comparaison avec d'autres nominations à travers les liens contextuels. Or, cette régularité générale « se réfracte » diversement dans la parole, selon toute une série de conditions situationnelles. Particulièrement dans la langue russe, le démonstratif ou le possessif est préférable pour la représentation réitérée du référent (facteur sémantique), à condition que le substantif se trouve dans une des positions syntaxiques «faibles », en qualité de complément circonstanciel, complément prépositionnel, attribut ou groupe structuralement isolé du prédicat (facteur syntaxique) [Volf, 1974 : 149].

On sait que la compatibilité des substantifs avec les déterminants dépend du degré d'individualisation, puisque les substantifs ne s'associent pas en contexte neutre aux possessifs et démonstratifs. En même temps, il est remarquable que les unités nominatives individualisantes se combinent facilement avec ces derniers dans les constructions affectives qui transmettent diverses nuances d'une modalité subjective. En effet, notre analyse montre que le déterminatif devant l'anthroponyme monoréférentiel prend une valeur émotivo-évaluative: les nominations onymiques de ce type ont le plus souvent des connotations négatives, exprimant des sentiments dévalorisants tels que éloignement, hostilité, désapprobation, indignation, aversion, haine, mépris, colère, etc. :

Moi, ça ne me regarde pas, Dieu merci. Vous, il faut vous consoler en songeant qu'il en a toujours été ainsi. Aucun homme à femmes n'y a échappé, pas même votre cher Casanova [MATZNEFF G., Ivre du vin perdu, 1981 : 17].

Un soir, le débat atteignit même de la hauteur : le sens de la vie, le pourquoi du mal. Le père Froissard allant jusqu'à citer Dostö̈evski: et penché vers Boris et moi :

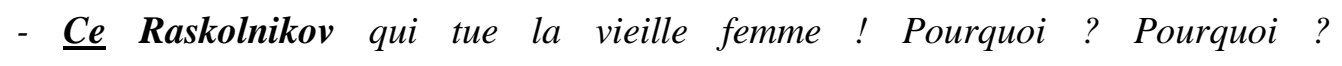
Ses courts membres s'agitaient sur le fauteuil [SCHREIBER B., Un silence d'environ une demi-heure, $1996: 246]$.

\section{Unité propriale «dé-capitalisée »}

La majuscule n'est pas un indice obligatoire ni une marque identificatrice des $\mathrm{Npr}$, contrairement à l'opinion de nombreux linguistes [Sag et Wasow, 1999], même dans le cadre de 
l' «eurocentrisme » onomastique, où elle se présente comme «un moyen assez efficace » [Vaxelaire, $2005:$ 74-75].

Pour M.-N. Gary-Prieur, c'est une propriété de langue, «si on s'en tient aux [Npr] prototypiques », qui « ne relève pas du libre-choix de celui qui écrit, comme semble le penser M. Wilmet» [1991: 96]. En fait, la majuscule dans la langue est conditionnée par les règles grammaticales et l'usage socio-institutionnel, mais d'autre part il ne faut pas nier la créativité littéraire et la volonté individuelle de l'auteur. La flexibilité linguistique majuscule/minuscule fait l'appel à la réinterprétation analytique et, par suite, au réinvestissement du sens d'une lexie.

Par exemple, W. Mańczak suppose que les Dupont doit être écrit en toute langue avec la majuscule [1968: 208]. Néanmoins, dans les cas d'antonomase, de Npr métaphoriquement employé, de communisation ou généralisation, lexicalisation, multiplication, abstraction et la variante les dupont(s) est très probable.

Vaxelaire a bien rappelé que la majuscule pour les Npr, selon Brunot [1965: 39], et l'article devant les Npr, selon Nyrop [1925 : 190], sont « relativement récents » [2005, 32-33]. Frontier prétend que « [il] suffit d'une majuscule pour transformer un Nc en Npr » [1997: 27]. Mais la majuscule ne transforme rien, elle est la marque et le résultat de la transformation.

\section{Unité propriale flexionnelle}

On constate habituellement que les Npr n'ont pas de flexions morphologiques [Sørensen, 1958 : 136] ou ont une flexion fixe [Togeby, 1982 : 120], sauf ceux qui n'existent qu'au pluriel comme pluralia tantum et désignent non pas un individu, mais un ensemble particulier.

Les uns disent que la singularité onymique est conditionnée par ce que le Npr transmet «a concrete and definite notion » [ibid. : 70], les autres l'expliquent par sa non-connotativité, d'où le parallèle avec l'emploi autonyme des Nc [Hewson, 1972: 89]. Or, l'usage des unités propriales dans la langue naturelle autorise des formes plurielles :

- référents homonymes (There are a few Johns in my class, Il y a quelques Jean(s) dans та classe, Eсть несколько Иванов в моем классе) ;

- emplois métaphoriques (They are the Hercules, Ce sont les Hercules, Они настоящие Геракльи);

- emplois du pluriel générique/familial (The Lawrences are leaving town, Les Lawrence sont en train de quitter la ville, Лоуренсы покидают город).

Les Npr au pluriel sont différemment traités par les linguistes. Les uns pensent qu'ici a lieu le passage du Npr au Nc [Kałuza, 1981 : 18], les autres disent qu'une référentialité singulière, qui leur est propre, est maintenue dans les emplois de ce genre [Superanskaja, 1983 : 13].

L'emploi métaphorique du $\mathrm{Npr}$ reste également discutable. C'est un procédé stylistiquement marqué, mais le sens sémiotique de son fonctionnement «symbolique » est traité de façon différente. La plupart des chercheurs y associent toujours le passage des Npr aux Nc. Or, une minorité admet un fonctionnement hybride (onyme - lexie) des unités bifonctionnelles [Semenova, 1990]. Donc, le singulier n'est pas un indice obligatoire des Npr, leur orthographe au pluriel est indécise. 


\section{Unité propriale interculturalisée}

La plupart des Npr étant ethnoculturels et ethnocentriques, c'est dans des conditions spécifiques qu'un Npr peut devenir relativement interculturel. La déonymisation est aussi un phénomène transculturel. Par exemple, les Fritz sont les soldats allemands pour les français et les russes à la fois, alors que les Bosch signifient les soldats allemands pour les Français, mais non pour les Russes. Les Npr sont plus exposés aux influences des facteurs extralinguistiques. Le Npr emprunté s'adapte aux normes et aux règles de la langue d'entrée en se pliant au façonnage phonologique et morphologique. Ainsi, les Fritz en russe se prononce et s'écrit sans article et avec la désinence du pluriel : fritz[y]. L'image de Fritz dans la culture populaire allemande correspond à la celle de Toto en français ou à celle de Vovočka en russe (diminutif de Vladimir) - « lest », « rusé », « retors » ou « naïf » et « vétilleux ».

\section{Unité propriale diminutive}

Il faut noter aussi l'abondance des variantes émotivo-stylistiques, qui véhiculent divers contenus connotatifs des noms personnels. Par exemple, dans le Dictionnaire des noms personnels russes, N. Petrovskij cite 40 diminutifs du nom le plus courant, celui d'Ivan (Vanja, Vanjka, Vanjuša, Vanëk, Vano, Vanječka, Ivaška, Ivanuška) et plus de 50 formes pour Olga (Olen'ka, Olečka, Olja, Oljuška, Olčik, Olgunčik, Lëlik, Lëlja). Aucun Nc ne produira autant de variantes à suffixes diminutifs ou hypocoristiques (Ivanišče). La diversité des formes connotatives, dans la nomination onomastique, crée la richesse des moyens pour exprimer des nuances émotivo-expressives. Ces moyens sont très nombreux pour les noms personnels, moins nombreux pour les surnoms et noms de famille, et assez rares pour les toponymes. Par exemple : Jojo - Joseph, Joël; Fifi - Philippe; Gérard - Gégé; Isabelle - Isa ; Elisabeth - Elise, Babette.

\section{Conclusion}

On peut en conclure que le fonctionnement des déterminants avec l'antonomase en Français et en Russe est réglé par des régularités communes. Toutefois, la détermination syntaxique de l'antonomase française remplit des fonctions distinctives. Si les démonstratifs et possessifs ont un rôle individualisant (en assurant la bifurcation référentielle) et affective (en accentuant la composante stylistique), les articles explicitent toujours la sémantique généralisée du Npr polyréférentiel, en soulignant le caractère qualificatif de cette dernière. En effet, même quand l'article précède l'antonomase, la nomination anthroponymique indique non l'identifiabilité des référents, mais l'identité des caractéristiques des individus comparés l'un à l'autre.

Dans les pays post-soviétiques, surtout la Russie, l'Ukraine, la Biélorussie, le Kazakhstan et, à l'Ouest, l'Europe et l'Amérique du Nord, un travail gigantesque a été effectué dans le domaine de l'onomastique théorique et descriptive. Malheureusement, nous ne connaissons pas toujours les ouvrages des uns des autres: les travaux des onomasticiens russophones ne sont pas connus à l'Ouest et, chez nous, on est informé par fragments des «performances» de l'onomastique occidentale. Dans le contexte de la globalisation scientifique, les barrières 
linguistiques et l'incompatibilité de la terminologie spécifique freinent le progrès commun. Pour atteindre l'intercompréhension entre les chercheurs et les écoles, nous avons besoin d'un travail théorique, comparatif et terminologique sérieux avec la mobilisation des onomasticiens slaves et européens.

\section{Bibliographie}

Allerton D.J., 1987, The Linguistic and Sociolinguistic Status of Proper Names // Journal of Pragmatics, Vol.11, no 1, pp. 61-92.

Boër S.E., 1978, Attributive names // Notre Dame J. Formal Logic, Vol. 19, Number 1, pp. 177-185.

Bonnard H., 2001, Les trois logiques de la grammaire française, Louvain-la-Neuve, Duculot, $251 \mathrm{p}$.

Brunot F., 1965 [1905-1937], Histoire de la langue française des origines à 1900. Paris : Colin.

Burge T.,1973, Reference and Proper Names, The Journal of Philosophy, LXX, 70: 425439.Chomsky N., 1975, Reflections on Language, New York, Pantheon Books, 269 p.

Curme J., 1955, A Grammar of the English language // Prace filosoficke faculty Brneske university.

Damourette J. \& Pichon E., 1911-1927 [1968], Des Mots à la pensée. Essai de grammaire de la langue française, Paris : d'Artrey.

Droste F.G., 1975, On Proper Names // Leuvense Bijdragen, Vol.64, nº 1, pp. 1-14.

Dubois J., 1965, Grammaire structurale du français : nom et prénom, Paris. Larousse.

Feoktistov A.P., 1969, O formakh vyraženija opredelennosti naricatel'nykh i sobstvennykh imen // Onomastika. Moskva, pp. 239-244.

Flaux N., 2000b, Nouvelles remarques sur l'antonomase, Lexique 15, pp. 117-144.

Frontier A., 1997, La Grammaire du français, collection "Sujets", Berlin.

Gary-Prieur M.-N., 1994. Grammaire du nom propre. Paris : Le Seuil.

Gary-Prieur M.-N., 1995, Les Simenon, Frédéric Dard et autres Japrisot » : l'effet du pluriel sur l'interprétation exemplaire du nom propre // Noailly M. (éd.), Nom propre et Nomination, Paris : Klincksieck, pp. 247-258.

Gary-Prieur M.-N., 1996, Figurations de l'individu à travers différentes constructions du nom propre // Cahiers de praxématique 27, pp. 57-73.

Jonasson K., 1990, Métaphores in absentia et la lexicalisation des noms propres // Actes du XI congrès des romanistes scandinaves, Trondheim 13/17 août 1990, Trondheim, pp. 261271.

Jonasson K., 1994, Le nom propre - Constructions et interprétations, Louvain-la-Neuve, Duculot, 256 p.

Hewson J., 1972, Article and noun in English. The Hague-Paris: Mouton, 137 p.

Kałuza H., 1981, The use of articles in contemporary English, Heidelberg, J. Groos, p. 86.

Kleiber G., 1981, Problèmes de référence : descriptions définies et noms propres, Metz, Centre d'Analyse Syntaxique, 538 p.

Kleiber, G. 1996. Noms Propres et Noms Communs: un Problème de Dénomination // Méta. XLI(4), pp. 567-589.

Kramsky J., 1972, The Article and the Concept of Definiteness in Language, Mouton, The Hague.

Leroy S., 2004, De l'identification à la catégorisation. L'Antonomase du nom propre en français, Louvain - Paris - Dudley MA : Peeters. 
Leroy S., 2005, Noms propres : la modification // Langue Française 146, Larousse, pp. 83-98.

Mańczak W., 1968, Le nom propre et le nom commun, Revue Internationale d'Onomastique 203, pp. 205-218.

Martin R. 1987, Langage et croyance : les « univers de croyance » dans la théorie sémantique, Mardaga.

Meyer B., Balayn J.-D., 1981, «Autour de l'antonomase de nom propre », Poétique 46, pp. 183-199.

Noailly M., 1987, Le nom propre en français contemporain : logique et syntaxe en désaccord imparfait // Cahiers de grammaire, $\mathrm{n}^{\mathrm{o}}$ 12, pp.65-78.

Noailly M., 1999, La Querelle des Noms Propres // Modèles linguistiques XX (1), pp. 107-112.

Nyrop K., 1925, Grammaire Historique de la langue française, 6 vols. Copenliagen: Gyldendalske Boghandel.

Pamp B., 1985, Ten theses on proper names // Names Vol.33 : 3, pp. 111-118.

Pulgram E., 1954, Preparation for Language Teaching // Applied Linguistics in Language Teaching. Ed. Ernst Pulgram. Washington, DC: Georgetown University Press. pp.75-85.

Sag, I.A. et Wasow. T., 1999, Syntactic Theory: an introduction . Stanford : CSLI Publ.

Searle J.R., 1969, Speech Acts. An Essay in the Philosophy of Language. Cambridge: Cambridge University Press.

Sloat C., 1969, Proper Nouns in English // Language 45, pp. 26-30.

Sørensen H.S., 1958, Word-classes in Modern English - with special reference to proper names, with an introductory theory of grammar, meaning and reference, Copenhague, p. 189.

Superanskaja A.V., 1973, Obミ̌aja teorija imeni sobstvennovo, Moscou, 345 p.

Superanskaja A.V., 1983, Struktura imeni sobstvennogo. Moskva.

Togeby K., 1982, Grammaire française Vol. I: Copenhague, Ak. Forlag, 551 p.

Van Langendonck W., 1985, Pragmatics and Iconicity as Factors Explaining the Paradox of Quantified Proper Names // Names, Vol.33, n 3, pp. 119-126.

Vasiljuk F.E., 1993, Struktura obraza // Voprosy psikhologii, № 5, pp. 5-19

Vaxelaire J.-L., 2005, Les noms propres - Une analyse lexicologique et historique. Paris, Honoré Champion, $953 \mathrm{p}$.

Vendler Z., 1967, Linguistics in philosophy. Ithaca NY: Cornell University Press.

Volf E.M., 1974, Grammatika i semantika mestoimenij. Moscou : AN URSSS, 223 p.

Wilmet M., 1986, La détermination nominale. Paris, P.U.F.

Wilmet M., 1991, Nom propre et ambiguïté // Langue française, № 92, pp. 113-124. 